\title{
Dedução do limite de Chandrasekhar: uma abordagem didática dos trabalhos originais do autor
}

\author{
Derivation of the Chandrasekhar limit: a didactic approach of the author's original papers \\ Edgard de F. D. Evangelista*10 \\ ${ }^{1}$ Instituto Nacional de Pesquisas Espaciais, Divisão de Astrofísica, São José dos Campos, SP, Brasil
}

Recebido em 04 de Junho, 2018. Revisado em 05 de Setembro, 2018. Aceito em 08 de Outubro, 2018.

\begin{abstract}
Os atuais modelos de evolução estelar estabelecem que estrelas possuindo até cerca de oito vezes a massa do Sol dão origem a anãs brancas no final de suas vidas. Na década de 1920 surgiram trabalhos que descreviam tais objetos como formados essencialmente por um gás de elétrons e núcleons, de forma que a força de sua pressão de degenerescência equilibrava a contração gravitacional, originando corpos estáveis e de alta densidade. Na década de 1930 Subrahmanyan Chandrasekhar lançou sua abordagem ao problema das anãs brancas em artigos onde ele deduziu as densidades e a massa máxima permitida para essas estrelas. Tal valor máximo para a massa é hoje conhecido como limite de Chandrasekhar e o objetivo aqui é analisar os trabalhos originais de seu descobridor, mostrando de forma didática os passos que levaram à dedução desse importante resultado.
\end{abstract}

Palavras-chave: Astrofísica, Anãs Brancas, Limite de Chandrasekhar

\begin{abstract}
The current stellar evolution models establish that stars which have up to approximately eight times the solar mass yield white dwarfs at the end of their lifes. Works appeared at the 1920s described such objects as formed essentially by a gas of electrons and nucleons, where the force of its degeneracy pressure equalized the gravitational contraction, giving rise to stable bodies with high densities. At the 1930s Subrahmanyan Chandrasekhar launched his approach to the white dwarf problem in papers where he deduced the densities and the maximum mass allowed for those stars. Such a maximum value is known nowadays as the Chandrasekhar limit and the aim here is to analyze the original papers by its discoverer, presenting in a didactic form the steps which led to the deduction of that important result.
\end{abstract}

Keywords: Astrophysics, White Dwarfs, Chandrasekhar Limit

\section{Introdução}

A partir dos modelos de evolução estelar sabe-se que estrelas possuindo até cerca de oito vezes a massa do Sol terminam suas vidas como anãs brancas, caracterizadas por densidades de $\sim 10^{6} \mathrm{~g} \mathrm{~cm}^{-3}$ e sendo compostas em sua maioria por um núcleo de carbono e oxigênio envolto em uma fina camada de hidrogênio e hélio [1. Essas densidades extremamente elevadas são explicadas pelo fato de no interior de uma anã branca não haver reações nucleares gerando energia para contrabalançar a contração gravitacional, de forma que ela se contrai até que a força gravitacional seja equilibrada pela força da pressão de degenerescência dos elétrons presentes no núcleo de tal estrela [2. Tal pressão exercida pelos elétrons é um efeito quântico e será discutido na Seção 2 .

O físico britânico Ralph H. Fowler propôs, em trabalho publicado em 1926 [3], que as anãs brancas são formadas por um gás de elétrons e núcleons não relativísticos (ou seja, onde as partículas têm velocidades muito menores do que a da luz) e não interagentes. Essa foi a primeira vez que a estatística quântica de Fermi-Dirac, a qual

*Endereço de correspondência: edgard.freitas.diniz@gmail.com. descreve o comportamento coletivo de partículas de spin semi-inteiro 4], foi aplicada a estudos de estrutura estelar. Posteriormente, o também britânico Edmund C. Stoner empregou o trabalho de Fowler para estudar as propriedades físicas das anãs brancas e derivou uma equação de estado para a matéria formadora de tal estrela, deduzindo valores máximos para sua massa e densidade [5,6].

No início da década de 1930 o físico indiano Subrahmanyan Chandrasekhar, já conhecendo os trabalhos de Fowler e Stoner, propôs sua própria solução para o problema da determinação das massas e densidades de anãs brancas. Ele publicou suas ideias em artigos $[7,8,8$ nos quais utiliza a solução da equação de equilíbrio hidrostático para estrelas esféricas e sem rotação em conjunto com a equação de estado para um gás de elétrons. Em [7], onde o autor utilizou a versão não relativística da equação de estado, é apresentada a estimativa para a densidade de uma anã branca; por outro lado, em $[8]$ é considerado o caso ultrarrelativístico da equação de estado (ou seja, o cenário onde a velocidade dos elétrons é muito próxima à da luz), fornecendo como resultado o famoso limite de Chandrasekhar, o qual estabelece a massa máxima possível para uma anã branca. 
É fato que diversos livros-texto de astrofísica e astronomia abordam o limite de Chandrasekhar, como por exemplo 1, 2, 9, 10. No entanto muitos apenas o mencionam de passagem ou apresentam demonstrações que, embora satisfatórias para especialistas e estudantes portadores de conhecimentos avançados, podem fugir ao alcance de um aluno cursando os semestres (ou períodos) iniciais da graduação em física. Nesse sentido o presente artigo pode ser interessante pois traz as deduções diretamente de sua fonte original de forma didática, exigindo do leitor apenas conhecimentos básicos em cálculo e mecânica quântica, além de noções de termodinâmica e relatividade especial, acessíveis em quaisquer livros-texto de física fundamental universitária, tais como 11 13; para cálculo, veja por exemplo 14. As deduções apresentadas aqui são interessantes também por colocarem o leitor em contato com o raciocínio original de Chandrasekhar.

Na Seção 2 é discutida a pressão de degenerescência dos elétrons, sendo deduzida sua expressão para os casos não relativístico e ultrarrelativístico; na Seção 3 calcula-se a densidade dos elétrons para a matéria estelar em função da densidade de massa e da fração de hidrogênio presente no meio; a Seção 4 aborda o artigo de Chandrasekhar onde é estimada a densidade típica de uma anã branca; a Seção 5 trata de outro artigo do referido autor, no qual ele deduz seu famoso limite. Finalmente, na Seção 6 são apresentadas as conclusões.

\section{Cálculo da pressão exercida por um gás de elétrons}

Seja um gás de elétrons livres confinados em dada região de volume fixo. Sobre as fronteiras de tal volume haverá uma pressão exercida por esse gás que não se origina da repulsão coulombiana entre os elétrons e nem é de natureza termodinâmica; a pressão em questão é de caráter puramente mecânico quântico, sendo consequência do princípio da exclusão de Pauli, o qual estabelece que duas partículas de spin semi-inteiro não podem ter simultaneamente o mesmo conjunto de números quânticos [4].

Se a região for considerada como um poço infinito tridimensional retangular de dimensões $l_{x}, l_{y}$ e $l_{z}$ cujo potencial obedece às condições de fronteira

$$
V= \begin{cases}0 & \text { se } 0<x<l_{x}, 0<y<l_{y}, 0<z<l_{z} \\ \infty & \text { fora do poço, }\end{cases}
$$

então a equação de Schrödinger independente do tempo para um elétron livre de massa $m$ preso nesse poço tem a forma

$$
-\frac{\hbar^{2}}{2 m} \nabla^{2} \psi=E \psi
$$

e possui como solução as funções de onda (veja, por exemplo, 4. para uma dedução detalhada):

$$
\psi_{p q r}=\sqrt{\frac{8}{p q r}} \operatorname{sen}\left[\frac{p \pi}{l_{x}} x\right] \text { sen }\left[\frac{q \pi}{l_{y}} y\right] \text { sen }\left[\frac{r \pi}{l_{z}} z\right],
$$

cujas respectivas energias são

$$
E_{p q r}=\frac{\hbar^{2} \pi^{2}}{2 m}\left[\frac{p^{2}}{l_{x}^{2}}+\frac{q^{2}}{l_{y}^{2}}+\frac{r^{2}}{l_{z}^{2}}\right] \text { onde } p, q, r \in \mathbb{N}^{*}
$$

Além disso, se definirmos as componentes do vetor de onda para o elétron como

$$
k_{x}=p \pi / l_{x}, k_{y}=q \pi / l_{y}, k_{z}=r \pi / l_{z},
$$

sendo sua magnitude dada por $k^{2}=k_{x}^{2}+k_{y}^{2}+k_{z}^{2}$, então $E_{p q r}$ pode ser escrita na forma

$$
E_{p q r}=\frac{\hbar^{2} k^{2}}{2 m} .
$$

Seja agora um espaço abstrato de eixos ortogonais $k_{x}$, $k_{y}$ e $k_{z}$. Supondo que essas coordenadas podem assumir apenas os valores discretos dados na Eq. (5), um elemento de volume em tal espaço é calculado como

$$
\begin{aligned}
& \Delta k_{x} \Delta k_{y} \Delta k_{z}= \\
& \frac{\pi}{l_{x}}[(p+1)-p] \frac{\pi}{k_{y}}[(q+1)-q] \frac{\pi}{k_{z}}[(r+1)-r]= \\
& \frac{\pi^{3}}{l_{x} l_{y} l_{z}}=\frac{\pi^{3}}{V},
\end{aligned}
$$

onde $V=l_{x} l_{y} l_{z}$ é o volume do poço e cada elemento dado pela Eq. (7) é associado a um estado distinto do elétron, caracterizado por valores específicos de $p, q$ e $r$.

Considere que dentro do poço haja $N$ elétrons livres, comportando-se como um gás. Em princípio esperariase que todos eles se acomodassem no estado de mais baixa energia, onde $p=q=r=1$. Porém, de acordo com o princípio da exclusão de Pauli, no máximo dois elétrons podem ocupar simultaneamente cada estado, já que esses podem ter apenas dois valores $(-1 / 2$ e $1 / 2)$ para o número quântico de spin.

Como $N$ em geral é muito grande, ou seja, da ordem do número de Avogadro, o volume total ocupado pelos elétrons no espaço $\left(k_{x}, k_{y}, k_{z}\right)$ pode ser calculado com boa precisão tratando-se essa região como o octante de uma esfera situado em $k_{x}>0, k_{y}>0$ e $k_{z}>0$ e cujo raio é identificado como o valor máximo de $k$. Assim, como cada par de elétrons ocupa um estado de volume $\pi^{3} / V$, tem-se

$$
\frac{1}{8}\left(\frac{4}{3} \pi k_{\max }^{3}\right)=\frac{N}{2}\left(\frac{\pi^{3}}{V}\right) .
$$

Da Eq. (8) deduz-se imediatamente que

$$
k_{\max }=\left(\frac{3 N \pi^{2}}{V}\right)^{1 / 3}
$$


A energia total do gás de elétrons é calculada por

$$
E_{\mathrm{tot}}=\int_{0}^{k_{\max }} d E
$$

onde

$$
d E=\frac{\hbar^{2} k^{2}}{2 m} d n
$$

é obtida multiplicando-se a energia dada pela Eq. (6) pelo número $d n$ de estados contidos em uma camada de espessura $d k$ do octante da esfera. O volume de tal camada é dado pela diferenciação do lado esquerdo da Eq. (8) e ignorando-se o subscrito em $k$, ou seja, é igual a $(1 / 8) 4 \pi k^{2} d k$. Além disso, considerando-se que cada estado possui volume dado pela Eq. (7) e é ocupado por dois elétrons, tem-se

$$
d n=\frac{2\left[(1 / 2) \pi k^{2} d k\right]}{\pi^{3} / V}=\frac{V}{\pi^{2}} k^{2} d k .
$$

Substituindo-se a Eq. (9), a Eq. (11) e a Eq. (12) na Eq. 10, chega-se a

$$
E_{\mathrm{tot}}=\frac{\hbar^{2} V}{2 \pi^{2} m} \int_{0}^{k_{\max }} k^{4} d k=\frac{\hbar^{2}\left(3 \pi^{2} N\right)^{5 / 3}}{10 \pi^{2} m} V^{-2 / 3} .
$$

A pressão $P$ exercida pelo gás de elétrons pode ser calculada aplicando-se a Eq. 13 à expressão termodinâmica 15

$$
P=-\left(\frac{\partial E}{\partial V}\right)_{N}
$$

o que fornece, ignorando-se o sinal negativo e introduzindose a densidade numérica de elétrons $n_{e}=N / V$ :

$$
P=\frac{\hbar^{2}}{5 m}\left(3 \pi^{2}\right)^{2 / 3} n_{e}^{5 / 3} .
$$

A Eq. 15 é normalmente conhecida como pressão de degenerescência ou pressão de exclusão [4] e é válida apenas para elétrons não relativísticos.

Em regime ultrarrelativistico, ou seja, quando os elétrons têm velocidades $v \approx c$ sendo $c$ a velocidade da luz, a pressão de degenerescência pode ser obtida de forma semelhante à utilizada na Eq. (15) mas tendo em mente que em tal regime a energia de cada elétron é dada por $E \approx p c$, onde $p$ é o momento 13 . Uma vez que $p=\hbar k$, tem-se

$$
E=\hbar c k \text {. }
$$

Utilizando-se a Eq. (16) ao invés da Eq. (6) para calcular $d E$, obtém-se

$$
d E=(\hbar c k) d n,
$$

$$
E_{\mathrm{tot}}=\frac{\hbar c V}{\pi^{2}} \int_{0}^{k_{\max }} k^{3} d k=\frac{\left(3 \pi^{2}\right)^{4 / 3}}{4 \pi^{2}} \hbar c N^{4 / 3} V^{-1 / 3}
$$

a aplicação da Eq. (18) à Eq. (14) leva imediatamente a

$$
P=\frac{\left(3 \pi^{2}\right)^{4 / 3}}{12 \pi^{2}}(\hbar c) n_{e}^{4 / 3} .
$$

As Eq. (15) e Eq. (19) podem ser demonstradas de maneira mais formal e rigorosa por meio da estatística quântica de Fermi-Dirac. Veja, por exemplo, [16]. Essas expressões valem para gases completamente degenerados, ou seja, onde todos os estados abaixo de um $k_{\max }$ estão ocupados por elétrons. Além disso, por simplicidade o regime ultrarrelativístico será referido de agora em diante apenas como regime relativístico.

\section{Cálculo de $n_{e}$ para o material estelar}

Para que as expressões deduzidas na Seção 2 sejam realmente úteis em suas diversas aplicações, é necessário que se possa determinar $n_{e}$ para cada caso. Aqui será demonstrado como obter $n_{e}$ em função da densidade de massa $\rho$ para um material estelar composto predominantemente por hidrogênio.

Seja $X$ a fração de massa de hidrogênio do material em relação à massa total. A densidade numérica de átomos de tal elemento é então calculada como 17

$$
n_{\mathrm{H}}=\frac{\rho}{m_{\mathrm{H}}} X
$$

com $m_{\mathrm{H}}$ representando a massa do átomo de hidrogênio. Como cada um desses átomos contribui com um elétron, então a densidade de elétrons correspondente é dada também pela Eq. 20. Para os outros elementos presentes no material, em geral o número de elétrons no átomo é aproximadamente metade do número de núcleons, de forma que a contribuição deles para $n_{e}$ é dada aproximadamente por 17

$$
n_{e}^{\text {outros }}=\frac{(1-X) \rho}{2 m_{\mathrm{H}}} .
$$

A expressão para $n_{e}$ é obtida somando-se as Eq. (20) e Eq. (21):

$$
n_{e}=\frac{\rho}{2 m_{\mathrm{H}}}(1+X)=\frac{\rho}{\mu_{e} m_{\mathrm{H}}},
$$

na qual a grandeza

$$
\mu_{e}=\frac{2}{1+X}
$$

é definida como o peso molecular médio dos elétrons.

De acordo com [18], há uma maneira diferente de se definir $\mu_{e}$, a saber:

a qual é a versão ultrarrelativística da Eq. (11). Considerandose a Eq. (9), a Eq. (12) e a Eq. (17) na Eq. (10), fica-se com

$$
\mu_{e}=\left[\sum_{A} c_{A} \frac{Z}{A}\right]^{-1}
$$


válida para a matéria totalmente ionizada. Na Eq. (24), $c_{A}$ é a fração em massa de átomos de massa atômica $A$ e número atômico $Z$ do material em relação à massa total, de forma que $\sum c_{A}=1$. Por exemplo, para o hidrogênio $(\mathrm{A}=1,008$ e $\mathrm{Z}=1)$ e o chumbo $(\mathrm{A}=207,2$ e $\mathrm{Z}=82)$ puros, a Eq. 24 fornece $\mu_{e}=1,01$ e $\mu_{e}=2,53$, respectivamente.

\section{A densidade das estrelas anãs brancas}

Em 1931 Chandrasekhar publicou o artigo intitulado The density of white dwarf stars, ou 'a densidade das estrelas anãs brancas', em português. Ele apareceu originalmente no The London, Edinburgh, and Dublin Philosophical Magazine and Journal of Science (atual Philosophical Magazine), no volume 11, número 70 e páginas 592-596. Posteriormente esse artigo foi reproduzido no Journal of Astrophysics and Astronomy [7] por ocasião do Jubileu de Diamante da Academia Indiana de Ciências.

Nesse trabalho o autor calcula a densidade típica das anãs brancas. Para isso ele considera uma estrela composta essencialmente de um gás completamente degenerado de elétrons não relativísticos e que está sujeita, em princípio, às pressões da radiação e de degenerescência desse gás, cujas forças equilibram a atração gravitacional. Porém, a partir desse ponto são consideradas duas simplificações: a pressão da radiação é desprezível (pois, ao contrário do que ocorre em estrelas como o Sol, no interior de uma anã branca não há fusão nuclear gerando energia); e a pressão de degenerescência é devida exclusivamente aos elétrons, uma vez que se considera que as outras partículas presentes no material (prótons e outros núcleos atômicos mais pesados) não estejam em estado degenerado 17. Assim, a pressão total que equilibra a contração devida à gravidade é dada pela Eq. [15.

Para $n_{e}$ é usada a expressão

$$
n_{e}=\frac{\rho}{\mu_{e} m_{\mathrm{H}}(1+f)}
$$

onde $f$ é a razão do número de íons para o número de elétrons. Chandrasekhar desprezou o valor de $f$, fazendo com que a Eq. 25. coincida com a Eq. 22. Substituindose a Eq. 22 na Eq. 15, obtém-se

$$
P=\frac{\hbar^{2}}{5 m}\left[\frac{3 \pi^{2}}{\mu_{e}^{5 / 2} m_{\mathrm{H}}^{5 / 2}}\right]^{2 / 3} \rho^{5 / 3} .
$$

Os íons considerados na definição de $f$ referem-se a prótons e núcleos mais pesados. Assim, em dado material estelar que seja formado principalmente por elementos pesados (na época se acreditava que as estrelas fossem compostas majoritariamente por elementos pesados) e que esteja completamente ionizado, haverá em geral dezenas de elétrons para cada íon, explicando o baixo valor de $f$ e a razão por tal quantidade ser desprezada nos cálculos.
A Eq. (26) é um caso particular das equações de estado características de polítropos ${ }^{1}$ as quais possuem a forma geral

$$
P=\kappa \rho^{\gamma}
$$

onde $\gamma=1+1 / n$ é o coeficiente adiabático, $n$ é o índice politrópico e $\kappa$ é uma constante de proporcionalidade [17].

Tratando-se a estrela como uma massa de gás isolada, esférica e sem rotação sendo mantida coesa pela sua própria atração gravitacional [20] e considerando que a pressão $P$ e a densidade $\rho$ em cada ponto de seu interior obedecem à relação politrópica dada pela Eq. (27), então a massa $M$ e o raio $R$ de tal esfera de gás relacionam-se por 20.

$$
\left(\frac{G M}{M^{\prime}}\right)^{n-1}\left(\frac{R^{\prime}}{R}\right)^{n-3}=\frac{[(n+1) \kappa]^{n}}{4 \pi G},
$$

onde $M^{\prime}$ e $R^{\prime}$ são constantes adimensionais com valores específicos para cada valor de $n$ ou $\gamma$ e podem ser encontradas em tabelas. Essas tabelas são apresentadas em 20,21] e contêm uma série de parâmetros (entre os quais $M$ e $R^{\prime}$ ), obtidos a partir de soluções numéricas da equação diferencial para o interior estelar.

Comparando-se a Eq. 27) com a Eq. (26), tem-se

$$
\kappa=\frac{\hbar^{2}}{5 m}\left[\frac{3 \pi^{2}}{\mu_{e}^{5 / 2} m_{\mathrm{H}}^{5 / 2}}\right]^{2 / 3},
$$

e $n=3 / 2$. Com isso, a Eq. 28 fornece

$$
M R^{3}=\left(\frac{3 \pi}{4}\right)^{2}\left[\frac{5 \hbar^{2}}{10 m G}\right]^{3} \frac{M^{\prime} R^{\prime 3}}{\mu_{e}^{5} m_{\mathrm{H}}^{5}} .
$$

Chandrasekhar utilizou valores correspondentes a $n=$ $3 / 2$ para $M^{\prime}$ e $R^{\prime}$ apresentados em 21, ou seja, $M^{\prime}=$ 2,7176 e $R^{\prime}=3,6571$. Substituindo-se os valores numéricos de tais constantes na Eq. (30) e usando $G=$ $6,6726 \times 10^{-8} \mathrm{~cm}^{3} \mathrm{~g}^{-1} \mathrm{~s}^{-2}, \hbar=1,0546 \times 10^{-27} \mathrm{erg} \mathrm{s}^{-1}$, $m=9,1094 \times 10^{-28}$ g e $m_{\mathrm{H}}=1,6733 \times 10^{-24} \mathrm{~g}$, porém deixando $\mu_{e}$ em aberto, obtém-se

$$
M R^{3}=\frac{4,3076 \times 10^{61}}{\mu_{e}^{5}} \mathrm{~g} \mathrm{~cm}^{3} .
$$

Considerando-se a anã branca como uma esfera de raio $\mathrm{R}$, sua densidade é calculada por $\rho=M /\left(4 \pi R^{3} / 3\right)$. Eliminando-se $R$ na Eq. (31) por meio da expressão para $\rho$, chega-se à densidade da anã branca:

$$
\rho=5,5421 \times 10^{-63}\left(\mu_{e}^{5} M^{2}\right) \mathrm{g} \mathrm{cm}^{-3} .
$$

Em astrofísica é usual que as massas das estrelas sejam dadas em unidade de massa solar, simbolizada por $\mathrm{M}_{\odot}$ e com valor dado por [1] $1,99 \times 10^{33} \mathrm{~g}$. Assim, $M$ na Eq. 32

\footnotetext{
${ }^{1}$ Um polítropo é uma esfera de gás mantida em equilíbrio por sua própria força gravitacional e com a propriedade de que a pressão em todos os pontos de seu interior obedece à Eq. 27. É usado em astrofísica como uma forma simplificada de modelo estelar 19.
} 
pode ser dada em $\mathrm{M}_{\odot}$ multiplicando-se e dividindo-se o lado direito de tal equação por $\left(1,99 \times 10^{33} \mathrm{~g}\right)^{2}$, fornecendo

$$
\rho=2,1947 \times 10^{4} \mu_{e}^{5}\left(\frac{M}{M_{\odot}}\right)^{2} \mathrm{~g} \mathrm{~cm}^{-3} .
$$

Note que $\rho$ na Eq. 33 ainda é dada $\mathrm{em} \mathrm{cm}^{-3}$ pois a transformação para $\mathrm{M}_{\odot}$ afeta apenas $M$. Além disso Chandrasekhar considerou $\mu_{e}=2,52^{2}$, de forma que a Eq. (33) se converte em

$$
\rho=2,143 \times 10^{6}\left(\frac{M}{M_{\odot}}\right)^{2} \mathrm{~g} \mathrm{~cm}^{-3} .
$$

O autor aplicou a Eq. (34) ao caso da estrela Sirius B. Desde que na época se acreditava que sua massa fosse de $0,85 \mathrm{M}_{\odot}$, a Eq. (34) fornece nesse caso $\rho_{\text {Sirius B }}=$ $1,548 \times 10^{6} \mathrm{~g} \mathrm{~cm}^{-3}$, o qual é cerca de 30 vezes superior ao valor aceito na época para a densidade dessa estrela (ou seja, $5 \times 10^{4} \mathrm{~g} \mathrm{~cm}^{-3}$ ). Essa discrepância foi justificada interpretando-se o valor calculado como sendo apenas o limite máximo para o caso em questão, correspondendo ao cenário hipotético onde o gás é totalmente degenerado. Ainda de acordo com o autor, tal configuração seria extremamente improvável na prática.

Segundo 17, o valor utilizado atualmente para $\mu_{e}$ em uma anã branca é de $\approx 2$. Se tal valor tivesse sido considerado no caso de Sirius B, o autor teria chegado a $5,074 \times 10^{5} \mathrm{~g} \mathrm{~cm}^{-3}$, o qual é cerca de três vezes inferior ao valor correspondente a $\mu_{e}=2,5$.

Hoje aceita-se que a massa e a densidade média de Sirius B sejam de respectivamente $\approx 1,0 \mathrm{M}_{\odot} \mathrm{e} \approx 3,0 \times$ $10^{6} \mathrm{~g} \mathrm{~cm}^{-3}$ 1, 9. Com isso e $\mu_{e}=2,0$, a Eq. (33) fornece $\rho_{\text {Sirius B }}=7,023 \times 10^{5} \mathrm{~g} \mathrm{~cm}^{-3}$. Esta quantidade e a calculada por Chandrasekhar são inferiores à densidade atualmente aceita de Sirius B por fatores de 0,23 e 0,52, respectivamente. Não há problemas em relação a esses números, uma vez que a Eq. (33) não deve ser considerada como um limite superior para a densidade, e sim como um valor típico estimado sob condições ideais. É fato que o autor interpretou sua estimativa de $\rho_{\text {Sirius B }}$ como o valor máximo possível para esse caso em particular, porém deve-se compreender que ele estava apenas justificando de forma fisicamente plausível seu resultado.

\section{A massa máxima de anãs brancas ideais}

O título desta Seção é a tradução para o português de The maximum mass of ideal white dwarfs, sendo este o título do artigo escrito por S. Chandrasekhar e publicado em 1931 no Astrophysical Journal, no volume 74, número 1 e páginas 81-82. Assim como [7], este trabalho foi

\footnotetext{
${ }^{2} \mathrm{O}$ autor usou o valor de $\mu_{e}$ aproximadamente igual ao do chumbo pois, conforme mencionado anteriormente, acreditava-se que o material estelar fosse composto essencialmente por elementos pesados. Veja a Eq. 24 e a discussão subsequente.
}

republicado no Journal of Astrophysics and Astronomy 8 .

Chandrasekhar propõe aqui uma questão interessante: o que se poderia obter caso fosse utilizada a fórmula relativística da pressão dada pela Eq. $(19)$ ao invés da Eq. 15 em um problema semelhante ao abordado na Seção 4 ?

Da mesma forma que a Eq. (15), a Eq. (19) tem a forma politrópica dada pela Eq. (27), porém agora $\kappa$ tem a forma

$$
\kappa=\frac{\hbar c}{12 \pi^{2}}\left[\frac{3 \pi^{2}}{\mu_{e} m_{\mathrm{H}}}\right]^{4 / 3},
$$

e $\gamma=4 / 3$ (ou $n=3$ ). Aplicando-se esse valor de $n$ à Eq. 28, obtém-se

$$
\left(\frac{G M}{M^{\prime}}\right)^{2}=\frac{(4 \kappa)^{3}}{4 \pi G}
$$

Substituindo a Eq. 35 na Eq. (36) e isolando $M$, chegase a

$$
M=\frac{M^{\prime}}{2} \frac{(3 \pi)^{1 / 2}}{\left(\mu_{e} m_{\mathrm{H}}\right)^{2}}\left[\frac{\hbar c}{G}\right]^{3 / 2} .
$$

De acordo com $\left[20, M^{\prime}=2.015\right.$ para $n=3$. Substituindose essa quantidade na Eq. (37), além dos valores das constantes $G, \hbar, m$ e $m_{\mathrm{H}}$ usados na Seção 4 obtém-se finalmente o valor numérico para $M$ :

$$
M=\frac{1,141 \times 10^{34}}{\mu_{e}^{2}} \mathrm{~g},
$$

o qual pode ainda ser colocado em termos de $\mathrm{M}_{\odot}$ dividindose a Eq. 38 por $1,99 \times 10^{33} \mathrm{~g}$ :

$$
M=\frac{5,734}{\mu_{e}^{2}} \mathrm{M}_{\odot} .
$$

Da mesma forma que na Seção 4 o autor considerou $\mu_{e}=$ 2,5 , fornecendo $M \approx 0,92 \mathrm{M}_{\odot}$. No entanto, tomando-se o valor aceito atualmente, ou seja, $\mu_{e}=2,0$, chega-se finalmente a

$$
M \approx 1,43 \mathrm{M}_{\odot} .
$$

O resultado dado pela Eq. 40 corresponde ao caso de uma estrela onde todos os elétrons de seu material são completamente degenerados, de forma que ele pode ser considerado como a máxima massa possível para uma anã branca. Eis o célebre limite de Chandrasekhar.

\section{Conclusões}

O propósito do presente artigo era analisar dois artigos de Subrahmanyan Chandrasekhar nos quais ele calcula a densidade e a massa máxima de estrelas anãs brancas, de forma a tornar esses importantes trabalhos acessíveis a estudantes da graduação em física, bastando que estes possuam conhecimentos básicos de cálculo e mecânica 
quântica, além de noções de termodinâmica e relatividade especial.

Inicialmente apresentou-se a dedução para a equação de estado de um gás de elétrons nos casos relativístico e não relativístico. A seguir, como ingrediente para as deduções subsequentes, foi mostrado como se obter a densidade numérica de elétrons do material estelar em função da densidade de massa e da fração de hidrogênio presente.

Subsequentemente foi discutido como Chandrasekhar estimou a densidade das anãs brancas, tratando o material estelar como um gás de elétrons totalmente degenerado não relativístico. Com a equação de estado para esse caso aplicada à relação entre massa e raio de uma estrela politrópica, chegou-se a $\approx 2,143 \times 10^{6}\left(M / M_{\odot}\right)^{2} \mathrm{~g} \mathrm{~cm}^{-3}$. Para o caso particular de Sirius $B$, tal resultado forneceu $1,548 \times 10^{6} \mathrm{~g} \mathrm{~cm}^{-3}$, o qual é cerca de 30 vezes superior ao valor aceito na época para essa estrela. O autor justificou essa diferença afirmando que a quantidade calculada seria apenas o limite máximo para a densidade, obtida no cenário ideal onde os elétrons são completamente degenerados. Usando a massa atualmente aceita para Sirius B e $\mu_{e}=2,0$, a Eq. (33) fornece $\rho_{\text {Sirius B }}=7,023 \times 10^{5} \mathrm{~g} \mathrm{~cm}^{-3}$. Esse valor e o obtido por Chandrasekhar são inferiores à densidade considerada hoje em dia para tal objeto (ou seja, $\approx 3,0 \times 10^{6} \mathrm{~g} \mathrm{~cm}^{-3}$ ) por fatores de 0,23 e 0,52 .

Por meio de processo semelhante ao utilizado na determinação da densidade, porém considerando a forma relativística da equação de estado para o gás de elétrons, Chandrasekhar deduziu que o valor máximo possível para a massa de uma anã branca era de $\approx 0,92 \mathrm{M}_{\odot} . \mathrm{Na}$ determinação desse valor foi utilizado $\mu_{e}=2,5$, entretanto hoje em dia se considera $\mu_{e} \approx 2$ para o caso em questão, de forma que atualmente a massa máxima considerada para esse tipo de estrela é de cerca de $1,43 \mathrm{M}_{\odot}$. Embora esse valor possa ser obtido independentemente das deduções anteriores envolvendo as densidades, todas as análises mostradas neste artigo são pertinentes pois mostram a evolução do raciocínio do autor, além disso os resultados da Seção 4 têm sua própria importância.

A quantidade $1,43 \mathrm{M}_{\odot}$ passou a ser conhecida como limite de Chandrasekhar e contribuiu para que seu descobridor recebesse o Prêmio Nobel de Física de 1983, o qual foi na ocasião dividido com o físico americano William Alfred Fowler 3 cujos estudos sobre a formação de elementos químicos no universo são também de grande importância.

Cabe aqui uma observação final: o leitor que consultar os trabalhos originais descritos neste artigo perceberá que os valores numéricos apresentados naqueles são levemente diferentes dos calculados aqui. As discrepâncias devem-se aos valores das constantes $G, \hbar, m$ e $m_{\mathrm{H}}$ utilizadas, as quais naquela época eram conhecidas com precisão um pouco menor.

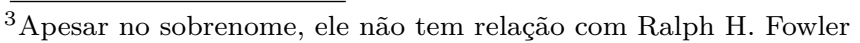
da referência 3 .
}

\section{Agradecimentos}

$\mathrm{O}$ autor agradece ao $\mathrm{CNPq}$ pelo apoio financeiro via bolsa PCI-DA (processo número 300887/2017-5). O autor agradece também ao INPE por toda a infraestrutura colocada à disposição.

\section{Referências}

[1] B.W. Carroll e D.A. Ostlie, An introduction to modern astrophysics (Addison-Wesley Publishing, Boston, 1996).

[2] M. Zeilik e S.A. Gregory, Introductory astronomy and astrophysics (Brooks/Cole, Pacific Grove, 1998), $4^{a}$ ed.

[3] R.H. Fowler, Mon. Not. R. Astron. Soc. 87, 114 (1926).

[4] D.J. Griffiths, Introduction to Quantum Mechanics (Cambridge University Press, Cambridge, 2017), $2^{a}$ ed.

[5] E.C. Stoner, Philos. Mag. 7, 63 (1929).

[6] E.C. Stoner, Philos. Mag. 9, 944 (1929).

[7] S. Chandrasekhar, J. Astrophys. Astr. 15, 105 (1994).

[8] S. Chandrasekhar, J. Astrophys. Astr. 15, 115 (1994).

[9] M. Harwit, Astrophysical Concepts (Springer, New York, 2000), $3^{a}$ ed.

[10] T. Padmanabhan, Theoretical Astrophysics: Stars and Stellar Systems (Cambridge University Press, Cambridge, 2000), v. II.

[11] P. Tipler e G. Mosca, Física para cientistas e engenheiros (Editora LTC, São Paulo, 2009), $6^{a}$ ed., v. 1, 2 e 3.

[12] D. Halliday, R. Resnick e J. Walker, Fundamentos de Física (Editora LTC, São Paulo, 2016), $10^{a}$ ed., v. 1, 2, 3 e 4.

[13] H.M. Nussenzveig, Curso de Física Básica (Editora Edgard Blücher, São Paulo, 2013), $5^{a}$ ed., v. 1, 2, 3 e 4

[14] M.A. Munem e D.J. Foulis, Cálculo (Editora LTC, São Paulo, 1982), v. 1 e 2.

[15] H.B. Callen, Thermodynamics: an introduction to the physical theories of equilibrium thermostatics and irreversible thermodynamics (John Wiley, New York, 1966).

[16] W. Greiner, L. Neise e H. Stöcker, Thermodynamics and statistical mechanics (Springer-Verlag, New York, 1995).

[17] A.R. Choudhuri, Astrophysics for Physicists (Cambridge University Press, Cambridge, 2010).

[18] E.C. Stoner, Mon. Not. R. Astron. Soc. 92, 651 (1932).

[19] K.S. Oliveira Filho e M.F.O. Saraiva, Astronomia e Astrofísica (Editora Livraria da Física, São Paulo, 2014), $3^{a}$ ed.

[20] A.S. Eddington, The internal constitution of the stars (Dover Publications, New York, 1959).

[21] R. Emden, Gaskugeln: Anwendungen der Mechanischen Wärmetheorie auf Cosmologische und Meteorologische Probleme (B.G. Teubner, Leizig and Berlin, 1907). 